\title{
The use of ketamine in a palliative-supportive care unit: a retrospective analysis
}

\author{
Sebastiano Mercadante ${ }^{1}$, Amanda Caruselli ${ }^{2}$, Alessandra Casuccio ${ }^{3}$ \\ ${ }^{1}$ Anesthesia and Intensive Care Unit and Pain Relief and Supportive-Palliative Care Unit, La Maddalena Cancer Center, Palermo, Italy; ${ }^{2}$ Regional \\ palliative care program, SAMO, Palermo, Italy; ${ }^{3}$ Department of Sciences for Health Promotion and Mother Child Care, University of Palermo, \\ Palermo, Italy \\ Contributions: (I) Conception and design: S Mercadante; (II) Administrative support: None; (III) Provision of study materials or patients: A Caruselli; \\ (IV) Collection and assembly data: A Casuccio; (V) Data analysis and interpretation: S Mercadante, A Casuccio; (VI) Manuscript writing: All authors; \\ (VII) Final approval: All authors. \\ Correspondence to: Sebastiano Mercadante, MD. Anesthesia \& Intensive Care Unit and Pain Relief \& Supportive Care, La Maddalena Cancer Center, \\ Via San Lorenzo 312, 90146 Palermo, Italy. Email: terapiadeldolore@lamaddalenanet.it or 03sebelle@gmail.com.
}

Background: To assess the response to ketamine in patients with difficult pain syndromes.

Methods: The charts of patients with uncontrolled pain despite opioid dose escalation of at least two opioids or a combination of them, selected for a burst of ketamine and midazolam were reviewed. One hundred $\mathrm{mg}$ /day of ketamine and midazolam $15 \mathrm{mg}$ /day by a continuous intravenous infusion for about 48 hours was offered to patients.

Results: Forty-four patients received a burst of ketamine. Ten patients did not achieve any improvement. Pain intensity decreased from a mean of 7.8 (SD, 1.6) to $2.8(\mathrm{SD}, 1.3)(\mathrm{P}<0.0005)$. The outcome was considered optimal, good, and mild in 24, 9, and 1 patients, respectively. Adverse effects attributable to ketamine did not have relevant intensity and no patient discontinued the treatment due to psychomimetic adverse effects.

Conclusions: This data suggests that a burst of ketamine and midazolam at low doses, may reverse an unfavourable opioid response, assisting the opioid switching. Although the role of ketamine remains controversial, it should not deter physicians to do not use that in specific conditions.

Keywords: Cancer pain; ketamine; refractory pain; adverse effects; palliative care

Submitted Jan 09, 2018. Accepted for publication Jan 12, 2018.

doi: 10.21037/apm.2018.01.01

View this article at: http://dx.doi.org/10.21037/apm.2018.01.01

\section{Introduction}

Ketamine in subanesthetic doses has been found to have analgesic effects. The analgesic effect of ketamine is likely to occur through its antagonism on $\mathrm{N}$-methyl-D-aspartate (NMDA) receptors (1). These receptors are involved in central sensitization, the development of hyperalgesia and allodynia in chronic pain, so these phenomena could be reversed by the binding of ketamine to these receptors (antihyperalgesic effect) (2). Its anti proinflammatory effects may be also responsible for its anti hyperalgesic effects. In the pathogenesis of pain, ketamine also acts by inhibiting the presynaptic neurons from the dorsal horns of the spinal cord through the release of excitatory substances such as glutamate and substance P. More recently, the role of ketamine in the activation of descending inhibitory pathways has been reported (3). Cancer pain is difficult to treat in some circumstances. Knowing that NMDA receptor changes are partially responsible for the development of hyperexcitability, there is a potential role of ketamine in the treatment of cancer pain as an adjuvant analgesic for refractory cancer pain, for neuropathic pain and for pain that no longer responds to high doses of opioids $(4,5)$. The use of Ketamine has been debated in 
terms of scientific evidence (6). In a recent multi-centric, dose-escalation, double-blind randomized, placebocontrolled clinical trial, a strong placebo effect and no additional clinic benefit for ketamine were found (7). However, patients' inclusion criteria, including a pain intensity of $\geq 3$ on a $0-10$ numerical rate scale, does not fit the clinical pattern commonly reserved for the use of ketamine. The aim of this study was to assess the response to ketamine in patients with difficult pain syndromes in a clinical setting of a supportive/palliative care unit (SPCU), where ketamine has been used for years to treat difficult pain conditions and reverse states of hyperexcitation.

\section{Methods}

The charts of patients who were admitted to a SPCU for a period of 28 months, from January 2013 to April 2016, were reviewed. It was not deemed necessary to ask for an approval from the ethical committee, given the retrospective nature of the study, reflecting a daily routine activity. A general written informed consent had been routinely given by patients for any therapeuthical intervention at admission. From this sample, patients who had received ketamine were selected.

Epidemiological characteristics were recorded, as well as pain mechanism, based on neurological examination performed by skilled physicians. Patients with uncontrolled pain despite opioid dose escalation of at least two opioids or a combination of them, were selected for a burst of ketamine and midazolam, according to local policy with a protocol used for several years: a burst of $100 \mathrm{mg} /$ day by a continuous intravenous infusion for about 48 hours was offered to patients who did not have a good response to opioid therapy, generally assisting a further opioid switching. In addition, midazolam was added at doses of $15 \mathrm{mg} /$ day for the same duration to prevent ketamineinduced psychomimetic effects, while maintaining an appropriate level of consciousness. Doses and duration of administration of these drugs were modified according to the clinical needs. Previous medications were maintained, unless for opioids. Changes in drugs and doses were flexible and dictated from the clinical situation. Similarly, opioids and their doses were changed individually according to the clinical response.

Pain intensity was recorded before ketamine administration (T0) and at discharge (on average 7 days after) (Tend). The discontinuation of treatment or the occurrence of relevant adverse effects to be attributed to the treatment were also collected from the charts (dreamlike state, uncontrolled eye movements, blurred vision, dizziness, unusual excitement, nervousness, or restlessness). Opioid-induced adverse effects with an intensity $\geq 2$ (on a scale 0 to 3 , where $0=$ absence, $1=$ mild intensity, $2=$ relevant intensity, $3=$ severe intensity), including nausea and vomiting, drowsiness, confusion, and so on, were scored, according to traditional monitoring report on a scale from 0 to 3. Data of opioid therapy and doses, expressed as oral morphine equivalents (OME) (8), were gathered from the charts at T0 and Tend. Patients who did not respond to this further treatment received alternative treatments, including intrathecal therapy, and were excluded from the analysis.

From the chart, the response was considered: optimal, when a $\geq 50 \%$ decrease in pain intensity was achieved with acceptable adverse effects; good when a $>30 \%$ decrease in pain intensity was achieved with acceptable adverse effects; mild when a decrease of less than $30 \%$ in pain intensity was achieved with acceptable adverse effects; negative, when no improvement of analgesia or inacceptable adverse effects when obtained within a week from starting ketamine.

\section{Statistical analysis}

Statistical analysis of quantitative and qualitative data, including descriptive statistics, was performed for all items. Continuous data were expressed as mean \pm standard deviation (SD), unless otherwise specified. Frequency analysis was performed using the Pearson's chi-square test and Fisher exact test, as needed. The paired samples student's $t$-test was used to compare pain intensity and opioid dosage, respectively, at the admission and discharge of the patients. Data were analyzed by IBM SPSS Software 22 version (IBM Corp., Armonk, NY, USA). All P values were two-sided and $\mathrm{P}<0.05$ was considered statistically significant.

\section{Results}

Forty-four patients received a burst of ketamine during the period taken into consideration (4.4\% of patients admitted to SPCU). The mean age was 54 years (SD, 11.6), and 19 patients $(43.2 \%)$ were males. Cancer diagnoses were in a rank order: urogenital $(n=14)$, lung $(n=12)$, gastrointestinal $(n=5)$, breast $(n=3)$, bone $(n=3)$, hematologic $(n=2)$, head and neck $(n=1)$, pancreas $(n=1)$, liver $(n=1)$, skin $(n=1)$, other $(n=1)$. Nineteen patients $(43.2 \%)$ were still 
Table 1 Numbers of patients receiving the different opioids (also in combination), at admission and at discharge

\begin{tabular}{|c|c|c|}
\hline Opioids & $\mathrm{n}$ & OME, mean [SD] \\
\hline \multicolumn{3}{|l|}{ Admission } \\
\hline $\mathrm{FE}$ & 5 & 625 [869] \\
\hline OX & 1 & 45 \\
\hline ME & 6 & 344 [233] \\
\hline ME, HY, TA & 1 & 235 \\
\hline ME, HY & 3 & 526 [336] \\
\hline $\mathrm{B}$ & 1 & 120 \\
\hline $\mathrm{B}, \mathrm{HY}, \mathrm{MO}$ & 2 & 375 [63] \\
\hline $\mathrm{B}, \mathrm{HY}$ & 2 & $400[0]$ \\
\hline TA, HY & 4 & 305 [145] \\
\hline HY & 3 & $300[28]$ \\
\hline $\mathrm{HY}, \mathrm{MO}$ & 1 & 710 \\
\hline $\mathrm{MO}$ & 2 & 325 [35] \\
\hline OX/NA & 3 & 57 [49] \\
\hline Total & 34 & 364 [379] \\
\hline \multicolumn{3}{|l|}{ Discharge } \\
\hline $\mathrm{FE}$ & 2 & $690[721]$ \\
\hline FE-ME & 1 & 285 \\
\hline ME & 7 & 332 [262] \\
\hline ME-TA & 5 & $463[221]$ \\
\hline ME, HY & 2 & $515[417]$ \\
\hline FE, MO & 1 & 240 \\
\hline $\mathrm{B}, \mathrm{HY}, \mathrm{MO}$ & 2 & $480[84]$ \\
\hline $\mathrm{B}, \mathrm{I}$ & 3 & $400[0]$ \\
\hline TA, HY & 2 & 320 [226] \\
\hline HY & 2 & 460 [480] \\
\hline TA & 1 & 200 \\
\hline MO & 5 & 522 [491] \\
\hline $\mathrm{MO}, \mathrm{ME}$ & 1 & 748 \\
\hline Total & 34 & 437 [304] \\
\hline
\end{tabular}

Doses (mean and SD) are expressed in mg as oral morphine equivalents (OME). FE, fentanyl; OX, oxycodone; ME, methadone; $\mathrm{HY}$, hydromorphone; $\mathrm{B}$, buprenorphine; $\mathrm{MO}$, morphine; TA, tapentadol. receiving chemotherapy. Ten patients did not achieve any improvement and were subsequently treated with further alternatives. Seven of these patients died in the subsequent days under palliative sedation for delirium. Opioid therapy and their doses, expressed in OME, at T0 and at Tend are presented in Table 1. Patients were receiving complex therapies, often with a combination of opioids, at high doses, unless for two patients ultimately receiving oxycodone, having a very narrow therapeuthic window.

Pain intensity was $7.8(\mathrm{SD}, 1.6)$ and $2.8(\mathrm{SD}, 1.3)$ at T0 and Tend, respectively. The difference was strongly significant $(\mathrm{P}<0.0005)$. According to the parameters described in methods, the outcome in these patients was considered optimal, good, and mild in 24,9 , and 1 patients, respectively. No differences in outcomes were observed for age $(\mathrm{P}=0.625)$, gender $(\mathrm{P}=0.107)$, and pain mechanism $(\mathrm{P}=0.113)$. Despite a slight increase, no statistical differences between T0 and Tend were reported in opioid doses, expressed as OME $(\mathrm{P}=0.997)$. The level of consciousness was acceptable, just requiring small changes in doses of ketamine and midazolam which were maintained at the same doses in most patients. Adverse effects attributable to ketamine did not have relevant intensity and were mild in intensity. No patient discontinued the treatment due to psychomimetic adverse effects.

\section{Discussion}

This retrospective review of patients with refractory pain syndromes who received a burst of ketamine provided relevant information from a clinical perspective. A strict selection criteria were adopted to test the efficacy of ketamine in very difficult cases, which represent a minority of patients admitted to a SPCU. Most of them, who had been previously treated with different types of opioids and combinations unsuccessfully, were potential candidates for interventional procedures, such as intrathecal analgesia (8). Ten patients discontinued the treatment, but most of them were closed to death, a phase in which most symptoms, including pain, are often overexpressed $(9,10)$. Indeed, according to response criteria adopted in this study, the majority of patients achieved an acceptable balance between analgesia and adverse effects and were discharged home with an opioid therapy at similar or slightly higher doses of opioids. This could be merely the result of advantages in conversion from one treatment to another one with opioid switching, given that burst ketamine was principally used to support opioid switching. Pain intensity in these patients 
dramatically decreased, allowing a discharge home on opioid therapy and some of them could continue their anticancer therapies. Of interest, feared adverse effects were not apparent with the concomitant use of midazolam, and did not required a discontinuation of therapy. Such approach, used for years at our institution, has been found to be safe and effective in a very selected population with difficult pain conditions not responsive to previous aggressive treatments.

Ketamine has been used for years for refractory cancer pain, although only few controlled studies have been published and evidence is not consistent (6). While most observational studies suggested that ketamine may be helpful in different clinical conditions, either decreasing pain intensity or opioid doses (10), in a recent well powered placebo-controlled study no advantages were found with the use ketamine that was also associated to toxicity (7). Similar findings were found in a smaller study, although $50 \%$ of patients seemed to have had major analgesic responses and no major adverse effects were reported (11). These findings contrast the benefits from ketamine observed in many open-label series $(10,12-20)$ even for prolonged periods of time $(21,22)$. The conclusions from this article have been contended, as they are valid only under the specific conditions and do not necessarily can applied to other uses of ketamine and patients' selection (22-24). There is no doubt that the power of study designed as placebocontrolled-randomized, and well numbered, is ideal and that there is a potential for observer bias and placebo responses in open-label trials. Indeed, these opposite outcomes could be attributed to methodological issues. Refractory pain was considered as a pain with an intensity of $\geq 3$, despite ongoing treatment with opioids and coanalgesics, without providing any attempt for optimization of opioid therapy, for example dose titration or switching. From a clinical perspective, patients with such characteristics are really common but not defined as refractory, as they could be easily treated without recurring to ketamine. The heterogeneity of patients was also of concern. Despite a median Karnofsky status of 60 , a large number of patients dropped out due to worsening conditions. Interestingly, the dose titration of ketamine to high doses, up to $500 \mathrm{mg} /$ day, is likely to produce expected central adverse effects. Potential cost savings with dissemination of the study results may have saved the Australian health care system approximately $\$ 663,000$ in annual hospitalisation costs (25). Of interest, while two-thirds of respondents reported practice change as a result of the randomised controlled trial, a minority remained convinced of the benefit of the drug from their own observations and would require additional evidence (26). This could reflect an inappropriate and widely disseminated use of ketamine possibly with the same indications reported in the Hardy's study. Taken together, data are not surprising, also considering that less than $50 \%$ of patients are responsive to ketamine test, with an effect lasting over drug discontinuation (27). Indeed, ketamine should be considered as an antihyperalgesic drug, rather than an analgesic to be given in normal conditions where minimal increases in opioid doses could be sufficient in most cases. Thus, results from this study cannot be applied to patients who failed previous aggressive treatments, possibly producing opioid-induce hyperalgesia $(3,28)$.

Even in patients who achieve a positive effect, the long-term use of ketamine is problematic, particularly for the use at home, given the characteristics of the drug and the possible limitations in availability in most countries. On the other hand, many physicians have been using the so-called burst ketamine approach (29). Several studies reported the lasting effect of a single ketamine infusion in patients with chronic pain receiving opioids. Ketamine was used in this manner in some open-label prospective audits of cancer patients which showed more than $50 \%$ of positive responses (13-20). This study, despite being retrospective, reflects the real world and years of experience in very selected patients who were unresponsive to previous treatment. Indeed, the differences between the results of Hardy et al. (7), and data from this study may also be explained not only by differences in patient selection and doses of ketamine, but overall by the "burst" modality and the prevention of psychomimetic adverse effects, which were easily covered by the concomitant administration of midazolam at doses unable to significantly reduce the level of consciousness. This procedure can produce extended periods of pain relief, acting by reducing the level of neuronal hyperexcitation, by reversing the level of opioid tolerance, as well improving analgesia (29). Adverse effects were limited and acceptable by using a combination of ketamine and midazolam at low doses, which were reduced only in minority of cases. Safety data confirm previous observations (11).

The principal limitation of this study is due to its retrospective nature, and the need to retrieve data from the charts. Assessment and documentation regarding symptom intensity and therapies, however, are regularly performed at this SPCU, regardless of a study participation, assuring a good quality of data. These data express exactly what 
happens in a SPCU where ketamine has been used for years to improve the analgesic response in difficult pain conditions. While these data do not provide evidence, they may suggest how to use ketamine, that is as a support for opioid switching, after failure of previous treatments. Symptomatic treatment, the use of coanalgesics, opioid switching, change of the route of administration are commonly used in cancer pain management, all of these being not evidence-based, and no randomized controlled trial may preclude the use of these modalities $(20,24,28)$.

In conclusion, this retrospective study suggests that a burst of ketamine, associated with doses of midazolam able to maintain the consciousness, may desensibilize states of hyperexcitation due to unfavourable opioid response, reversing tolerance and/ or hyperalgesia, facilitating and assisting the opioid switching. The role of ketamine is such complex and controversial that there is still incomplete evidence to state that ketamine is not of value in cancer pain $(30,31)$. Strict protocols of a randomized controlled trial often do not reflect the difficult conditions of a selected population, that are challenging and hardly resolved by a level of evidence (24). In situations where analgesic options have failed, ketamine could be a reasonable "third line"choice (31-33). Large and multicenter studies should confirm these data, as controlled studies in the contest of a very selected and difficult population are unlikely to provide information to be applied in the clinical setting.

\section{Acknowledgements}

None.

\section{Footnote}

Conflicts of Interest: The authors have no conflicts of interest to declare.

Ethical Statement: This is retrospective analysis of charts of patients who received treatment commonly given to patients admitted to the unit. A general written consent has been routinely obtains by patients for any therapeutical intervention at admission.

\section{References}

1. Fisher K, Coderre TJ, Hagen NA. Targeting the $\mathrm{N}$-methyl-d-aspartate receptor for chronic pain management. Preclinical animal studies, recent clinical experience and future research directions. J Pain Symptom Manage 2000;20:358-73.

2. Quibell R, Prommer EE, Mihalyo M, et al. Ketamine. J Pain Symptom Manage 2011;41:640-9.

3. Mao J, Price DD, Mayer DJ. Mechanisms of hyperalgesia and morphine tolerance: A current view of their possible interactions. Pain 1995;62:259-74.

4. Price DD, Mayer DJ, Mao J, et al. NMDA-recptor antagonists and opioid receptor interactions as related to analgesia and tolerance. J Pain Symptom Manage 2000;19:S7-11.

5. Mercadante S, Portenoy RK. Opioid poorly-responsive cancer pain. Part 3. Clinical strategies to improve opioid responsiveness. J Pain Symptom Manage 2001;21:338-54.

6. Bell RF, Eccleston C, Kalso EA. Ketamine as an adjuvant to opioids for cancer pain. Cochrane Database Syst Rev 2012;11:CD003351.

7. Hardy J, Quinn S, Fazekas B, et al. Randomized, doubleblind, placebo-controlled study to assess the efficacy and toxicity of subcutaneous ketamine in the management of cancer pain. J Clin Oncol 2012;30:3611-7.

8. Mercadante S, Ferrera P, Villari P, et al. Frequency, indications, outcomes, and predictive factors of opioid switching in an acute palliative care unit. J Pain Symptom Manage 2009;37:632-41.

9. de la Cruz M, Noguera A, San Miguel-Arregui MT, et al. Delirium, agitation, and symptom distress within the final seven days of life among cancer patients receiving hospice care. Palliat Support Care 2015;13:211-6.

10. Bredlau AL, Thakur R, Korones DN, et al. Ketamine for pain in adults and children with cancer: a systematicreview and synthesis of the literature. Pain Med 2013;14:1505-17.

11. WinegardenJ, CarrDB, Bradshaw YS. Intravenousketaminefor rapid opioid dose reduction, reversal of opioid-induced neurotoxicity, and pain control in terminal care: case report and literature review. Pain Med 2016;17:644-9.

12. Salas S, Frasca M, Planchet-Barraud B, et al. Ketamine analgesic effect by continuous intravenous infusion in refractory cancer pain: considerations about the clinical research in palliative care. J Palliat Med 2012;15:287-93.

13. Kotlińska-Lemieszek A, Luczak J. Subanasthetic ketamine: an essential adjuvant for intractable cancer pain. J Pain Symptom Manage 2004;28:100-2.

14. Fitzgibbon EJ, Viola R. Parenteral ketamine as an analgesic adjuvant for severe pain: development and retrospective audit of a protocol for a palliative care unit. J Palliat Med 
2005;8:49-57.

15. Jackson K, Ashby M, Martin P, et al. "Burst" ketamine for refractory cancer pain: an open-label audit of 39 patients. J Pain Symptom Manage 2001;22:834-42.

16. Jackson K, Ashby M, Howell D, et al. The effectiveness and adverse effects profile of "burst" ketamine in refractory cancer pain: The VCOG PM 1-00 study. J Palliat Care 2010;26:176-83.

17. Good P, Tullio F, JacksonK, et al. Prospective audit of short-term concurrent ketamine, opioid and antiinflammatory ('triple-agent') therapy for episodes of acute on chronic pain. Intern Med J 2005;35:39-44.

18. Mercadante S, Villari P, Ferrera P, et al. Opioid switching and burst ketamine to improve the opioid response in patients with movement-related pain due to bone metastases. Clin J Pain 2009;25:648-9.

19. Mercadante S, Villari P, Ferrera P. Burst ketamine to reverse opioid tolerance in cancer pain. J Pain Symptom Manage 2003;25:302-5.

20. Mercadante S, Arcuri E, Tirelli W, et al. Analgesic effect of intravenous ketamine in cancer patients on morphine therapy: a randomized, controlled, double-blind, crossover, double-dose study. J Pain Symptom Manage 2000;20:246-52.

21. Jackson K, Ashby M, Goodchild C. Subanestheticketamine for cancer pain: by insisting on level I/II evidence, do we risk throwing the baby out with the bath water?J Pain Symptom Manage 2005;29:328-30.

22. Leppert W. Ketamine in the management of cancer pain. J Clin Oncol 2013;31:1374.

23. Lossignol DA, Obiols-Portis M, Body JJ. Successful use of ketamine for intractable cancer pain.Support Care Cancer 2005;13:188-93.

Cite this article as: Mercadante S, Caruselli A, Casuccio A. The use of ketamine in a palliative-supportive care unit. A retrospective analysis. Ann Palliat Med 2018;7(2):205-210. doi: 10.21037/apm.2018.01.01
24. Mercadante S, Lodi F, Sapio M, et al. Long-term ketamine subcutaneous continuous infusion in neuropathic cancer pain. J Pain ymptom Manage 1995;10:564-8.

25. Jackson K, Franco M, William L, et al. Ketamine and cancer pain: the reports of my death have been greatly exaggerated. J Clin Oncol 2013;31:1373-4.

26. Mercadante S. Ketamine: to be or not to be. Ann Palliat Med 2013;2:37-9

27. McCaffrey N, Hardy J, Fazekas B. Potential economic impact on hospitalisations of the Palliative Care Clinical Studies Collaborative (PaCCSC) ketamine randomised controlled trial. Aust Health Rev 2016;40:100-5

28. Hardy JR, Spruyt O, Quinn SJ, et al. Implementingpracticechange in chroniccancerpain management: clinicianresponse to a phase III study of ketamine. Intern Med J 2014;44:586-91.

29. Cohen SP, Wang S, Chen L, et al. An intravenous ketamine test as a predictive response tool in opioidexposed patients with persistent pain. J Pain Symptom Manage 2009;37:698-708.

30. Franco M, William L, Poon P, et al. Critical appraisal of randomized controlled trials: the beginning of thought. J Pain Symptom Manage 2014;47:e1-2

31. Mak P, Broadbear JH, Kolosov A, et al. Long-Term Antihyperalgesic and Opioid-Sparing Effects of 5-Day Ketamine and Morphine Infusion ("Burst Ketamine") in Diabetic Neuropathic Rats. Pain Med 2015;16:1781-93.

32. Jonkman K, van de Donk T, Dahan A. Ketamine for cancer pain: what is the evidence? Curr Opin Support Palliat Care 2017;11:88-92.

33. Hocking G, Cousins MJ. Ketamine in chronic p-basedain management: an evidence-based review. Anesth Analg 2003;97:1730-9. 